\title{
Development of a Biomimetic semicircular canal with MEMS sensors to restore balance
}

\author{
Mohammad Amin Raoufi, Sajad A. Moshizi, Amir Razmjou, Shuying Wu, Majid Ebrahimi Warkiani, \\ Mohsen Asadnia, Senior Member, IEEE
}

\begin{abstract}
A third of adults over the age of 50 suffer from chronic impairment of balance, posture, and/or gaze stability due to partial or complete impairment of the sensory cells in the inner ear responsible for these functions. The consequences of impaired balance organ can be dizziness, social withdrawal, and acceleration of the further functional decline. Despite the significant progress in biomedical sensing technologies, current artificial vestibular systems fail to function in practical situations and in very low frequencies. Herein, we introduced a novel biomechanical device that closely mimics the human vestibular system. A microelectromechanical systems (MEMS) flow sensor was first developed to mimic the vestibular haircell sensors. The sensor was then embedded into a three-dimensional (3D) printed semicircular canal and tested at various angular accelerations in frequency range of $0.5 \mathrm{~Hz}$ to $3 \mathrm{~Hz}$. The miniaturized device embedded into a 3D model printed will respond to mechanical deflections and essentially restore the sense of balance in patients with vestibular dysfunction. The experimental and simulation studies of semicircular canal presented in this work will pave the way for the development of balance sensory system, which could lead to the design of a low-cost and commercially viable medical device with significant health benefits and economic potential.
\end{abstract}

Index Terms - Artificial semicircular canals, Auditory haircells, Piezoresistive flow sensors, Vestibular sensors

\section{INTRODUCTION}

$\mathrm{T}$ HE vestibular labyrinth in the inner ear is a receptor apparatus for sensing head motion and position, orienting the body in space, maintaining stability of the visual world, and modulating the autonomic nervous system. This delicate system is located in the inner ear and comprised of otolithic organs and three semicircular canals (SCCs) which measure the linear accelerations and rotational movements along three rotational axes (Yaw, Pitch and Roll), respectively [1].The vestibular system can be impaired by a myriad of reasons such as disease, injury, or aging, thereby giving rise to nausea, vertigo, oscillopsia, dizziness, and imbalance. There are a number of different conditions that affect the vestibular system, including benign paroxysmal positional vertigo, Meniere's disease (MD)

This work was supported by the Australian Research Council Centre Discovery Early Career Researcher Award (DECRA) DE180100688.

Mohammad Amin Raoufi, Sajad A. Moshizi and Shuying Wu, are with School of Engineering, Macquarie University, Sydney, New South Wales 2109, Australia.

Majid Ebrahimi Warkiani is with School of Biomedical Engineering, University of Technology Sydney, Sydney 2007, Australia. and acute vestibulopathy (vestibular neuritis), which causes difficulty in walking and maintaining balance for sufferers of the condition [2]. In the US it is estimated that $36 \%$ of the adults over the age of 40 suffer from chronic vestibular dysfunction [3]. This number increases with age reaching almost $50 \%$ for people over 60, with a 12 -fold increase in the risk of falling [3]. Estimates show that there have been roughly 500,000 patients with complete bilateral vestibular deficit (BVD) in Europe and the United State [4].

The causes of these diseases are not entirely known and this has led to mistreatments and has thus made evaluating the treatments much more difficult [5]. In order to tackle these inadequacies and find a robust way to analyze and treat balance impairments, researchers started to fabricate prosthetic vestibular systems. In the 1960 's, the idea of the first vestibular system arose and since then there has been a significant progress in this area. Notably, a research group from Harvard [6] performed the first implant vestibular system animal study which was then continued by a research team from Johns Hopkins [7]. Most of the previously developed vestibular implants are based on Cochlear implant technology in which the information related to the head angular acceleration around different rotational axis is detected by an accelerometer and transmitted to the brain using modulation of the rate of electrical impulses delivered to the vestibular neurons [8]. During the last decades, many attempts have been made to develop a vestibular implant for clinical trials [9]. The first breakthroughs in the development of vestibular implants in animals were the research works of Cohen and Suzuki [10-13]. In those studies conducted during the 1960s, eye movements and postural changes due to electrical stimulation of semicircular canal nerves in various animal models were systematically scrutinized. Following this, several animal studies have been conducted to design and implant synthetic vestibular systems in rodents such as guinea pigs [6], longtailed chinchillas [14], and some non-human primates such as squirrel monkeys [15], and rhesus monkeys [16, 17]. However, the applications of vestibular implants in human clinical trials has been hindered due to the complexity of sensor-based

Amir Razmjou is with UNESCO Centre for Membrane Science and Technology, School of Chemical Science and Engineering, University of New South Wales, Sydney 2052, Australia.

Mohsen Asadnia is with School of Engineering, Macquarie University, Sydney, New South Wales 2109, Australia (email: mohsen.asadnia@mq.edu.au). 
devices detecting head motion and the difficulty of transferring encoded signals to the ampullary nerves [18].

This prototype is a step forward for development of implantable vestibular systems that can be utilized as a prosthesis to provide cures for those suffering from various vestibular system impairments. Therefore, there is an immediate need for further investigation and research in this area.

Recently Microelectromechanical System (MEMS)-based sensors have drawn significant attention in hearing [19] and vestibular systems [20] due to their promising outcome for treatment and diagnosis of hearing and vestibular impairments. MEMS devices offer a miniaturised, low-cost and low-power sensing system [21] that can be integrated with other essential parts of an implant cochlear or vestibular system and provide a suitable platform for the development of biocompatible implant biomedical devices [22]. MEMS devices have been extensively used in design and fabrication of acoustic transducers in Cochlear implants [23-25] or basilar membrane [26, 27]. Moreover, the rapid development of MEMS-based sensors have propelled thorough grasp of body motion sensing [20, 28-30].

However, designing a proper MEMS sensory system has been hampered because of the complexity of this organ. Gong and Merfeld [31] developed a prototype semicircular canal prosthesis in which a piezoelectric vibrating gyroscope measures angular velocity of the head, and a digital filter is used to modulate the pulse rate of electrical stimulation. Shkel et al. [32] investigated a MEMS-based implantable prosthesis consisting of orthogonal triads of accelerometers and gyroscopes on-a-single fingernail sized chip to precisely sense, extract and transmit 3D motion. Afterwards, Shkel and Zeng [20] described development of a MEMS-based vestibular prosthesis prototype. They combined MEMS and integrated circuit technologies to shrink the sensor size, reduce the fabrication cost, and integrate the sensing-processingstimulating functions. Weinberg et al. [33] used unique algorithms using modestly performing micromachined gyroscopes and accelerometers for balance-impaired patients. Later on, Constandinou et al. [34] proposed an innovative design of an integrated circuit with a capacitive MEMS-based inertia sensor for attaining similar transfer characteristics to semicircular canal. Bhatti et al. [35] developed a fully implantable vestibular prosthesis for sensing the angular head rotations based on the biomechanics of the human semicircular canal. The results revealed that compared to the conventional gyroscopes, their detecting method can provide significant power savings. In another research effort, Andreu et al. [36] mimicked the natural vestibular semicircular canals' operation using a gyroscope manufactured via a commercially available MEMS process in which a sensing structure deforms due to fluid inertial force caused by rotation. In 2011, Chiang et al. [37] developed a multichannel vestibular prosthesis (MVP) to re-establish vestibular nerve activity. The system included a direct implantation in the labyrinth and motion sensors to monitor gravity, rotation, and acceleration. Dellea et al. [38, 39] thoroughly investigated the use of gyroscopes based piezoresistive Nano electromechanical system (NEMS) gauges in vestibular systems.

Utilizing an appropriate sensor in order to mimic haircells inside semicircular canals is the main issue in modelling the behavior of the natural organ. For this purpose, in the present work, a bio-mimetic flow sensor is proposed in which a membrane-based pressure sensor is used. Inspired by the cave fish lateral line haircell sensor, a low cost downsized piezoresistive sensor that uses a liquid crystal polymer (LCP) membrane-based pressure sensor has been developed. This sensor has interesting characteristics such as flexibility, sensitivity, cost effectiveness and robustness, and can be used in many different real-world applications [40].

This paper aims to use engineering analysis and advanced manufacturing techniques to develop an artificial semicircular canal and generate a fundamental understanding of the biological balance organ. We have used the numerical modelling to study the fluid dynamics of the vestibular system. MEMS piezoresistive sensors have been developed and packaged inside a 3D printed semicircular structure to mimic the biological balance organ. Head angular rotation was simulated by developing a rotating table that closely mimics human neck angular acceleration. Rotation causes the liquid inside the canal to flow, thereby deforming the sensor, which enables us to measure a corresponding response from the sensor. The main aim of this research is to fabricate a low-cost and viable medical implantable device to mimic the human balance sensory system, with the eventual goal of mitigation of vestibular system dysfunction in patients.

\section{FABRICATION OF ARTIFICIAL VESTIBULAR SYSTEM}

The fabrication process of the proposed vestibular system consists of two main parts, namely development of the MEMS piezoresistive haircell sensors, and 3D printing the vestibular structure. Essential criteria for haircell sensors are highsensitivity, ease of use, low-cost, biocompatibility and small size. The semicircular canal structure is designed to closely mimic the biological vestibular system.

\section{A. Development of the MEMS piezoresistive haircell sensors}

Liquid Crystal Polymer (LCP) has been used to develop the microdiaphragm for the haircell sensor. LCP, which is made up of aligned molecule chains with crystal-like spatial regularity, is a class of aromatic thermoplastic polymers, and demonstrates a highly ordered structural configuration in both the liquid and solid states [41]. The fabrication process and characterization of the MEMS piezoresistive sensor have been described elsewhere [42-45], however, for the sake of comprehensiveness, we provide an overview of the fabrication process here. Full characterization of the sensors in terms of fabrication process, response time, gauge factor calculation, and calibration plots have been presented in [40, 44, 46]. Figs. 1A-D illustrates the fabrication process. Commercially available LCP sheet (LCP 3908) with thickness of $25 \mu \mathrm{m}$ is used for the sensor development. In order to be able to deposit $\mathrm{Au}$ on the LCP sheet, the sheet needs to be temporarily bonded to a silicon wafer (Fig. 1A). So the first step of fabrication was 
to bond the LCP layer to a $400 \mu \mathrm{m}$ silicon layer using an intermediate adhesion layer of a $4 \mu \mathrm{m}$ thick AZ P4903, spincoated on both the silicon wafer and the LCP film. This step was followed by spin coating a $5 \mu \mathrm{m}$ photo-resist on LCP and patterning it for the lift-off process (Fig. 1B). Cr (20 nm) / Au $(100 \mathrm{~nm})$ are sputter-deposited, and the lift-off process performed to form gold piezoresistors (Fig. 1C). Following this, the whole wafer was kept in acetone for 10 minutes to remove the LCP layer from the silicon wafer. Fig. 1E shows an image of the sensor after fabrication process. Size of the diaphragm is $2000 \mu \mathrm{m}$.

To form the flow sensor, a non-conductive vertical 3D printed standing structure was mounted on the centre of the LCP membrane (Fig. 1F). The structure consists of a high-aspect ratio Accura Si60 polycarbonate polymer pillar with $350 \mu \mathrm{m}$ diameter and $2700 \mu \mathrm{m}$ height. The pillar was manually positioned at the centre of the LCP membrane and bonded using a micro-drop of a non-conductive epoxy (EPO-TEK H70E) followed by thermal curing at $70{ }^{\circ} \mathrm{C}$ for 10 hours. Whereas the root of the pillar is attached to the LCP sensing membrane, its distal apex extends into the external flow. The Accura Si60 polycarbonate plastic and LCP are used because of their resistance to humidity and moisture, and suitability for underwater applications. The complete sensor functions by flow passing over the sensor pillar in the semi-circular canal causing bending of the LCP membrane. When the rotational induced flow passes the polymer pillar, it is subject to unbalanced applied forces caused by the asymmetric shear and pressure forces on it due to the flow. The force on the pillar generates strain on the underlying LCP membrane to bend, causing a change in the resistance of the sensor, through which the flow-generated force is measured. Under no flow conditions, with the pillar fixed on the sensor, the strain gauges in the sensor have a no load resistance in the range of $650 \Omega$ and $1.2 \mathrm{k} \Omega$. The developed artificial haircell sensor was finally mounted on a $2 \mathrm{~mm}$ rebate at the centre of the 3D printed base using non-conductive epoxy (EPO-TEK H70E), forming the microdiaphragm. Table 1 lists physical properties of the LCP sensor.

\section{B. Fabrication of the lateral semicircular canal (LSCC)}

The biological semicircular canal is comprised of three nearly perpendicular lateral, anterior and posterior canals, used to measure head rotation in each of three dimensions. In this study, in order to simplify the fabrication process and downstream tests and to avoid complexity, a simplified model of the SCC containing only the lateral canal was used for experiments and further analysis. Fig. 2 shows the fabrication process of the lateral semicircular canal (LSCC) prostheses (based on the real human morphological data provided in Table 2). From the comprehensive study conducted by David et al. [47] and Kate et al. [48], the simplified morphology of SCCs can be defined by four main features, being the diameters of duct curvature, ampulla, utricle and slender duct, as depicted in Fig. 2A. Each semicircular canal in the human vestibular system can be distinguished by its own specific size. The values used for the semicircular canal fabrication and simulations represent the morphology of the LSCC (Table 2). Based on this morphological data, the LSCC was modeled in Solidworks software and saved as an STL file (Fig. 2A), and then printed using a Stereolithography apparatus (SLA) 3D printer. It should be noted that the dimensions were scaled up by a factor of 6.0 in order to facilitate the fabrication process and further packaging and analysis.

In order to package the artificial haircell sensor in the LSCC, the non-conductive epoxy (EPO-TEK H70E) was firstly used to attach the sensor to the base and the pillar to the centre of the LCP membrane, as shown in Fig. 2D. The thickness of 3D printed base is $2000 \mu \mathrm{m}$. After this, a conductive epoxy (Polytec EC 101) was used to connect the ultra-thin $(0.012 \mathrm{~mm}$ diameter $)$ copper wire to the sensor. The flow sensor was carefully inserted into the ampulla, and then the base was firmly anchored to the LSCC to make the final structure (Fig. 2C). Finally, the LSCC was filled with deionized water and mounted on a movable stage for testing. The deionized water (density and viscosity of $1 \mathrm{~g} / \mathrm{ml} 3$ and $1 \mathrm{cP}$ respectively) was used because of its chemical proximity to the endolymph (biological fluid contained in the semicircular canals) [49].

\section{EXPERIMENTAL SETUP}

Fig. 3 shows the different steps and equipment required to test and record the LSCC response to rotary stage movements. The rotary stage mimics head movements based on the morphological data provided in Table 3. Generally, head rotation can be decomposed to three axes, namely yaw, roll and pitch, with approximate maximum inclination of $80^{\circ}, 65^{\circ}$ and $40^{\circ}$ respectively. According to the Grossman et al. [50] study, the maximum frequency for head rotation around yaw and roll axes does not exceed $2.5 \mathrm{~Hz}$, whereas the maximum value for the pitch axis is $3.6 \mathrm{~Hz}$. The rotary stage used in this step has three separate axes of rotation, each capable of mimicking head rotation around a specific axis. This is implement with servo motors controlled by an Arduino UNO microcontroller integrated with an Adafruit pulse-width modulation servo shield, with task-specific code written in the Arduino IDE language (Fig. 3A). Table rotation causes the fluid inside the LSCC to flow and deflect the flow sensor (Fig. 3B). Resultant deflection in the LCP membrane changes the resistance of the strain sensor, which is converted to voltage through a Wheatstone-Bridge circuit, with the voltage measured by a National Instruments (NI) NI-9239 Data Acquisition (DAQ)[51] device (Fig. 3C). The measured data was analysed with NI LabVIEW Signal Express software to identify the output voltage amplitude (Fig. 3D). To reach a steady state condition, the rotary table was programmed to rotate for 5 cycles and then recording of data was undertaken. The stage movement was specified by rotation angle and frequency.

\section{DISCUSSION AND RESULTS}

In this section, the response of the sensor to table movements under various rotational conditions is numerically and experimentally investigated. To this end, firstly, a set of 
experiments were conducted to measure the sensor output with regards to the rotation axis, frequency and amplitude. Then, rotational movements of the LSCC were numerically modelled in Ansys Fluent 19 to better explain the experimental results by analyzing the flow inside the rotating LSCC. While the rotary table is capable of rotating over a wide range of frequencies and angles, test conditions should be compatible with real human head movement limits shown in Table 3. Through a study done by Ferrario et al. [52], the average angles for the full range of neck motion were obtained. These results were used to determine values for rotation around roll, pitch and yaw axes (Fig. 4A). As can be seen from Fig. 4, in the human inner ear the posterior, anterior and lateral canals are arranged almost perpendicularly in 3 dimensions. Movement of fluid inside the LSCC is mainly associated with rotation of the head around a yaw axis [47].

\section{A. Experimental results}

The sensor response to the table rotation for a wide range of angles and frequencies was studied comprehensively. The stage position information was recorded simultaneously with the sensor output as illustrated in Figs. 5A-D. As can be seen in Fig. 5 , for 60-degree rotation around the yaw axis and at all frequencies, the embedded sensor in the LSCC model has two peak signal values during each cycle. Interestingly, it was observed that one peak is always smaller than the other one, which has not been previously explored by other investigators. Since this behavior has not to our knowledge been mentioned in the literature, after repeating the experiments a number of times to ensure repeatability, it was postulated that this behavior must occur due to the asymmetric geometry of the LSCC, causing the hydrodynamic forces to act unequally on the sides of the sensor pillars. To test this idea, the system was modelled numerically, producing a similar signal pattern. As shown in Fig. 5, increasing the rotational frequency results in the peak voltage gradually increasing from $0.65 \mathrm{mV}$ at $0.5 \mathrm{~Hz}$ to $2.5 \mathrm{mV}$ at $1.3 \mathrm{~Hz}$. Due to the fluid inertia inside the canal, there is a phase difference between the table and sensor output voltage.

Fig. 6 shows the sensor responses to a variety of rotational and physiological conditions. As shown in Figs. 6A and 6B for rotation around the yaw axis, the sensor average peak-peak voltage gradually increases with frequency and rotation angle. This originates from the fact that by increasing the frequency and rotation angle, the fluid inside the canal has greater relative velocity to the sensor. In addition, in contrast to the higher frequency $(1.3 \mathrm{~Hz})$ case, tests conducted at lower frequency $(0.5 \mathrm{~Hz})$ have more uniform average peak-peak voltages for all the rotation angles. This is consistent with oscillation at lower frequency and angle inducing less energetic flow spread over longer time period, causing the LCP membrane to have minor reflections and produce lower voltage responses.

Due to the physiology of the human head, the semicircular canals are located on either side of the head rather than positioned in the centre of the rotational axis, so studying the sensor output for an off-centre position is important. Based on the morphological data [53], the semicircular canals are positioned approximately $4.5 \mathrm{~cm}$ away from the centre axis. Fig. 6B shows the sensor output of the off-centre rotation around the yaw axis for various table frequencies and 60-degree rotation. Similar to the situation in which the SCC is located precisely on the centre of the yaw axis, increasing the rotation frequency and angle increases the average peak-peak voltage values. However, for the off-centre case, the values are higher due to the greater distance between the canal and the centre of the rotation, which causes an increase in the fluid momentum inside the canal. Fig. 6C compares the sensor responses around yaw, roll and pitch axes under the condition of off-centre rotation. The obtained results illustrate that the rotation of the LSCC around the yaw axis causes the sensor to generate a higher peak-peak voltage than that of around roll and pitch axes. This is due to the fact that in yaw axis rotation, the fluid has a relatively open path to flow around the semicircular canal, but for the roll and pitch axes, the fluid rotation is substantially constrained. Finally, to evaluate how the geometry of the LSCC can affect the sensor output, two LSCCs with different utricle size were designed, one $10 \%$ larger in volume than the other. Fig. 6D illustrates that in general the sensor output slightly increases with the utricle size. Increasing the utricle volume augments the fluid motion inside the canal and consequently intensifies the flow and consequent force on the sensor.

\section{B. Numerical results}

In order to understand the flow dynamics inside the LSCC, measure the sensor response to the LSCC movement and analyze experimental results, the LSCC rotation was simulated numerically in Ansys Fluent 19 using a two-way fluid-structure interaction (FSI) method. The sensor pillar tip movement was measured in order to analyze the sensor output voltage, as the sensor output is directly related to the LCP membrane deflections caused by the pillar movements. The simulation was performed for a range of rotation frequencies and angles, similar to those in the physical experiments. As shown in Fig. 7, the system including LSCC, sensor pillar, LCP membrane and their base were separately modelled then assembled, enabling us to evaluate each part's response to the rotational movement individually. The entire system is assumed to rotate with the same angular velocity as the table. As shown in Fig. 7A, a non-uniform tetrahedral mesh was used for the semicircular channel, with denser mesh around the sensor. Also, a uniform hexahedral mesh was employed for the sensor pillar, LCP and the base. To meet the mesh independency criteria, a total of $180 \times 10^{3}$ elements were used for all the parts. As can be seen, a hole was embedded at the bottom of the base plate to allow the LCP membrane to easily bend when the pillar deflects due to the hydrodynamic forces (Figs. 7B-C). As the system rotated, the fluid flow inside the LSCC was measured by solving continuity and momentum (Navier-Stokes) equations as follow:

$\nabla . V=0$

$\partial V / \partial t+\rho(V . \nabla) V=-\nabla P+\mu \nabla \nabla^{2} V$

where $\rho$ and $\mu$ are the density and the viscosity of the deionized water respectively, and $\mathrm{P}$ is the static pressure. At each time 
step, after measuring the flow field, the pressure and wall shear stress were measured over the sensor pillar to evaluate pillar deflection and LCP bending. At each subsequent time step, the flow field was updated based on the new position of the pillar and the updated boundary conditions. The Navier-Stokes equations were solved using no slip boundary condition for the LSCC walls and solid-fluid interface for the surface of the sensor pillar. For structural analysis, the base was assumed to be fixed and had only rotational movement, LCP was attached to the base, and the pillar had fluid-solid interface boundary conditions allowing data transfer to the surrounding fluid. In accordance with the experimental results, the voltage peak values were always higher in the clockwise direction, so we concluded that the asymmetric geometry of the LSCC utricle and ampulla must play an important role in unequal distribution of hydrodynamic forces on the pillar surface. In other words, the complex geometry of the utricle and ampulla causes the maximum shear stress and pressure on each side of the pillar to differ for each half-cycle, causing the pillar to have a different maximum deflection in each rotational direction.

Fig. 8 shows the stream lines originated from 3D flow inside LSCC, and pressure and velocity contours on the symmetry plane around the pillar for the case of 60-degree amplitude and $1 \mathrm{~Hz}$ frequency for $\mathrm{t}=1.25 \mathrm{~s}$ and $\mathrm{t}=1.75 \mathrm{~s}$ corresponding to the table stationary positions during the its second cycle. As can be seen, the sudden expansion inside the utricle and ampulla reduces the total velocity values in these regions and causes secondary flows to form in the utricle region. This asymmetry in geometry and flow field causes the sensor to function directionally and enables the system to distinguish the direction of head rotation. This phenomenon can be explained by showing the fluid pressure around the sensor. It can be clearly seen that whereas maximum fluid pressure on the left hand side of the pillar at $\mathrm{t}=1.25 \mathrm{~s}$ is around $5 \mathrm{~Pa}$, at $\mathrm{t}=1.75 \mathrm{~s}$ the maximum pressure on the right hand side is approximately $2 \mathrm{~Pa}$, so the pillar deflection will differ in each direction. In addition, velocity vectors around the pillar show that while the LSCC structure stops rotating at $\mathrm{t}=1.25 \mathrm{~s}$ and $\mathrm{t}=1.75 \mathrm{~s}$, the fluid inside the canal is still flowing counterclockwise and clockwise respectively. This lag is caused by fluid inertia and the fluid's tendency to maintain its previous state. Since the pillar movement and consequently sensor output voltage are caused by the fluid motion inside the LSCC, there is a phase difference between the pillar displacement (or output voltage) and LSCC rotation. The maximum deflection of the pillar follows the maximum rotation of the table with the phase difference of $\phi=0.15 \mathrm{~s}$.

Fig. 9 shows the sensor tip displacement for various rotation angles and frequencies. Since the sensor output voltage is correlated to the deformation of the LCP membrane and the LCP deformation is related to the pillar movement, we decided to evaluate the experimental results by measuring the sensor tip displacement. Interestingly, the sensor tip movement showed the same pattern as the output voltage from the experiments. It can be seen that, under the same frequency, with increasing rotation angle the sensor deformation noticeably increases (Fig. 9A). For a 15-degree rotation, the sensor displacement is less than $0.05 \mu \mathrm{m}$, but when increasing the rotation angle to 60 degrees, the sensor tip displacement reaches $0.3 \mu \mathrm{m}$. This originates from the fact that increasing rotating angle allows a longer duration for the fluid inside the canal to flow, and consequently bending the pillar more substantially. In addition, by increasing the rotation angle, the peak to peak sensor displacement increases, which is consistent with the experimental results. It can be seen that whereas for 15-degree rotation and $1 \mathrm{~Hz}$ frequency peak-to-peak sensor displacement is similar at $<0.05 \mu \mathrm{m}$ in each direction, for 60 -degree rotation it increases to around $0.3 \mu \mathrm{m}$. Fig. 9B shows the sensor tip movement for different rotation frequencies and 60-degree table rotation. As can be seen, peak to peak displacement increases with increasing rotation frequency. This is due to acceleration force being strongly correlated to the frequency $(a=r w 2, w=2 \pi f$ ), so that increasing the frequency magnifies the force due to acceleration and consequently pillar displacement. Fig. 9C illustrates the pillar tip movement with respect to yaw, roll and pitch axes rotations. As can be seen, rotation around yaw-axis deflects the pillar much more than rotation around roll and pitch axes in the LSCC, since stronger flows form through yaw-axis rotation, and consequently affect the pillar more. The LSCC is almost 7 times more sensitive to yaw-axis rotation compared with roll-axis rotation, which shows while each SCC can sense rotations in all directions, each one is selective to one particular axis. Fig. 9D shows the sensor tip displacement for LSCC rotating both clockwise and anticlockwise. It can be seen that, apart from the initial rotation direction, the higher peak always occurs in the same direction.

\section{CONCLUSION}

In this work we used SLA 3D printing technology and piezoresistive sensors to fabricate a bionic inner ear organ, in order to analyze the function of the vestibular system. Based on morphological data, a simplified model of lateral semicircular canal (LSCC) was 3D printed and equipped with a novel piezoresistive flow sensor. This equipment was mounted on a table with the ability to rotate freely around yaw, roll and pitch axes in order to analyze the effects of table rotation on sensor response. Interestingly, the results showed that while our system uses a different sensing mechanism compared to human vestibular system haircell sensors, it is capable of sensitivity to the direction of rotation in such a way that the maximum sensor output always occurs in one direction. To better understand this behavior, the system was numerically modeled using the twoway FSI method in Ansys Fluent software. The results showed that the asymmetric geometry of the LSCC causes the flowinduced pressure on the pillar to be different from each direction, thereby having higher deflection in the sensor in a specific direction. Additionally, due to the fluid inertia inside the canal, a phase difference between the stage output and sensor output voltage was observed. It was also shown that by increasing the rotation frequency and angle the average peakpeak voltage increased. In addition, rotation along the roll axis resulted in lower sensor output than that of the yaw axis, since fluid movement within the LSCC was mainly associated with head rotation around the yaw axis. Moreover, average peak- 
peak voltage trends become more uniform for all rotation angles at low frequencies compared to those of high frequencies. Also, off-centre LSCC showed higher values of voltage under the same circumstances due to the greater distance between the centre of the rotation and the canal. Comparison of the sensor responses around pitch, yaw and roll axes showed that LSCC generates higher voltages around the yaw axis. Finally, by increasing the size of the utricle by $10 \%$, the sensor output slightly increased due to the formation of a stronger fluid flow inside the LSCC. In addition to experimental results, the sensor tip displacement in numerical results follows the same pattern as the sensor output voltage obtained in experimental results. Our results introduce new insights into the mechanisms of balancing in the human body and can be used to extend the application of piezoresistive sensors in modelling the vestibular systems.

\section{ACKNOWLEDGMENT}

This work was supported By the Australian Research Council Centre Discovery Early Career Researcher Award (DECRA) DE180100688. The Authors would like to thank Dr. Ajay Giri Prakash Kottapalli for his contribution in the device fabrication.

\section{REFERENCES}

[1] P. F. Smith, "Bionic balance organs: progress in the development of vestibular prostheses," The New Zealand Medical Journal (Online), vol. 130, pp. 5665, 2017.

[2] T. Brandt and M. Dieterich, "The dizzy patient: don't forget disorders of the central vestibular system," Nature Reviews Neurology, vol. 13, p. 352, 2017.

[3] Y. Agrawal, J. P. Carey, C. C. Della Santina, M. C. Schubert, and L. B. Minor, "Disorders of balance and vestibular function in US adults: data from the National Health and Nutrition Examination Survey, 2001-2004," Archives of internal medicine, vol. 169, pp. 938-944, 2009.

[4] B. K. Ward, Y. Agrawal, H. J. Hoffman, J. P. Carey, and C. C. Della Santina, "Prevalence and impact of bilateral vestibular hypofunction: results from the 2008 US National Health Interview Survey," JAMA otolaryngology-head \& neck surgery, vol. 139, pp. 803-810, 2013.

[5] C. A. Foster and R. E. Breeze, "Endolymphatic hydrops in Ménière's disease: cause, consequence, or epiphenomenon?," Otology \& Neurotology, vol. 34, pp. 1210-1214, 2013.

[6] W. Gong and D. M. Merfeld, "Prototype neural semicircular canal prosthesis using patterned electrical stimulation," Annals of biomedical engineering, vol. 28, pp. 572-581, 2000.

[7] C. D. C. Santina, A. A. Migliaccio, R. Hayden, T.-A. Melvin, G. Y. Fridman, B. Chiang, et al., "Current and future management of bilateral loss of vestibular sensation - an update on the Johns Hopkins Multichannel Vestibular Prosthesis Project,"
Cochlear implants international, vol. 11, pp. 2-11, 2010.

[8] R. F. Lewis, "Advances in the diagnosis and treatment of vestibular disorders: psychophysics and prosthetics," Journal of Neuroscience, vol. 35, pp. 5089-5096, 2015.

[9] D. M. Merfeld and R. F. Lewis, "Replacing semicircular canal function with a vestibular implant," Current opinion in otolaryngology \& head and neck surgery, vol. 20, pp. 386-392, 2012.

[10] B. Cohen and J.-I. Suzuki, "Eye movements induced by ampullary nerve stimulation," American Journal of Physiology-Legacy Content, vol. 204, pp. 347-351, 1963.

[11] B. Cohen, J.-I. Suzuki, and M. B. Bender, "XVI Eye Movements from Semicircular Canal Nerve Stimulation in the Cat," Annals of Otology, Rhinology \& Laryngology, vol. 73, pp. 153-169, 1964.

[12] J.-I. Suzuki, K. Goto, K. Tokumasu, and B. Cohen, "LXXII Implantation of Electrodes near Individual Vestibular Nerve Branches in Mammals," Annals of Otology, Rhinology \& Laryngology, vol. 78, pp. 815826, 1969.

[13] D. Bagger-Sjöbäck, A. Brodal, B. Cohen, G. Dohlman, J. Fredrickson, R. Gacek, et al., Vestibular System Part 1: Basic Mechanisms: Springer, 1974.

[14] C. C. Della Santina, A. A. Migliaccio, and A. H. Patel, "Electrical stimulation to restore vestibular function development of a 3-d vestibular prosthesis," in 2005 IEEE Engineering in Medicine and Biology 27th Annual Conference, 2006, pp. 7380-7385.

[15] D. M. Merfeld, C. Haburcakova, W. Gong, and R. F. Lewis, "Chronic vestibulo-ocular reflexes evoked by a vestibular prosthesis," IEEE transactions on biomedical engineering, vol. 54, pp. 1005-1015, 2007.

[16] J. O. Phillips, S. M. Bierer, L. Ling, K. Nie, and J. T. Rubinstein, "Real-time communication of head velocity and acceleration for an externally mounted vestibular prosthesis," in 2011 Annual International Conference of the IEEE Engineering in Medicine and Biology Society, 2011, pp. 3537-3541.

[17] C. Dai, G. Y. Fridman, N. S. Davidovics, B. Chiang, J. H. Ahn, and C. C. Della Santina, "Restoration of 3D vestibular sensation in rhesus monkeys using a multichannel vestibular prosthesis," Hearing research, vol. 281, pp. 74-83, 2011. J. S. Golub, L. Ling, K. Nie, A. Nowack, S. J. Shepherd, S. M. Bierer, et al., "Prosthetic implantation of the human vestibular system," Otology \& neurotology: official publication of the American Otological Society, American Neurotology Society [and] European Academy of Otology and Neurotology, vol. 35, p. 136, 2014.

[19] J. Jang, J. H. Jang, and H. Choi, "Biomimetic Artificial Basilar Membranes for Next-Generation Cochlear Implants," Advanced healthcare materials, vol. 6, p. 1700674, 2017. 
[20] A. M. Shkel and F.-G. Zeng, "An electronic prosthesis mimicking the dynamic vestibular function," Audiology and Neurotology, vol. 11, pp. 113-122, 2006.

[21] T. Islam and S. Mukhopadhayay, "Wearable sensors for physiological parameters measurement: physics, characteristics, design and applications," Wearable Sensors: Applications, Design and Implementation; Mukhopadhyay, SC, Islam, T., Eds, pp. 1-31, 2017.

[22] M. Parvez Mahmud, N. Huda, S. H. Farjana, M. Asadnia, and C. Lang, "Recent Advances in Nanogenerator-Driven Self-Powered Implantable Biomedical Devices," Advanced Energy Materials, vol. 8, p. 1701210, 2018.

[23] A. Gesing, F. Alves, S. Paul, and J. Cordioli, "On the design of a MEMS piezoelectric accelerometer coupled to the middle ear as an implantable sensor for hearing devices," Scientific reports, vol. 8, p. 3920, 2018.

[24] B. Ilik, A. Koyuncuoğlu, Ö. Şardan-Sukas, and H. Külah, "Thin film piezoelectric acoustic transducer for fully implantable cochlear implants," Sensors and Actuators A: Physical, vol. 280, pp. 38-46, 2018.

[25] M. Zurcher, D. Young, M. Semaan, C. Megerian, and W. Ko, "MEMS middle ear acoustic sensor for a fully implantable cochlear prosthesis," in 2007 IEEE 20th

International Conference on Micro Electro Mechanical Systems (MEMS), 2007, pp. 11-14.

[26] H. Jeon, J. Jang, S. Kim, and H. Choi, "Characterization of a Piezoelectric AlN Beam Array in Air and Fluid for an Artificial Basilar Membrane," Electronic Materials Letters, vol. 14, pp. 101-111, 2018.

[27] Y. Kim, J.-S. Kim, and G.-W. Kim, "A Novel Frequency Selectivity Approach Based on Travelling Wave Propagation in Mechanoluminescence Basilar Membrane for Artificial Cochlea," Scientific reports, vol. 8, p. 12023, 2018.

[28] H. Zeng and Y. Zhao, "Sensing movement: Microsensors for body motion measurement," Sensors, vol. 11, pp. 638-660, 2011.

[29] F. Ejeian, S. Azadi, A. Razmjou, Y. Orooji, A. Kottapalli, M. E. Warkiani, et al., "Design and applications of MEMS flow sensors: A review," Sensors and Actuators A: Physical, 2019.

[30] H. Khan, A. Razmjou, M. Ebrahimi Warkiani, A. Kottapalli, and M. Asadnia, "Sensitive and flexible polymeric strain sensor for accurate human motion monitoring," Sensors, vol. 18, p. 418, 2018.

[31] W. Gong and D. M. Merfeld, "System design and performance of a unilateral horizontal semicircular canal prosthesis," IEEE Transactions on Biomedical Engineering, vol. 49, pp. 175-181, 2002.

[32] A. Shkel, J. Liu, C. Ikei, and F.-G. Zeng, "Feasibility study on a prototype of vestibular implant using MEMS gyroscopes," in SENSORS, 2002 IEEE, 2002, pp. 1526-1531.

[33] M. S. Weinberg, C. Wall, J. Robertsson, E. O'Neil, K. Sienko, and R. Fields, "Tilt Determination in MEMS Inertial Vestibular Prosthesis," Journal of
Biomechanical Engineering, vol. 128, pp. 943-956, 2006.

T. G. Constandinou, J. Georgiou, and C. Toumazou, "A fully-integrated semicircular canal processor for an implantable vestibular prosthesis," in 2008 15th IEEE International Conference on Electronics, Circuits and Systems, 2008, pp. 81-84.

[35] P. T. Bhatti and M. A. McClain, "Low-power sensing for vestibular prostheses," in 2011 Annual

International Conference of the IEEE Engineering in Medicine and Biology Society, 2011, pp. 3131-3134.

[36] C. Andreou, Y. Pahitas, and J. Georgiou, "Bioinspired micro-fluidic angular-rate sensor for vestibular prostheses," Sensors, vol. 14, pp. 1317313185, 2014.

[37] B. Chiang, G. Y. Fridman, C. Dai, M. A. Rahman, and C. C. Della Santina, "Design and performance of a multichannel vestibular prosthesis that restores semicircular canal sensation in rhesus monkey," IEEE transactions on neural systems and rehabilitation engineering, vol. 19, pp. 588-598, 2011.

[38] S. Dellea, A. Longoni, G. Langfelder, A. Nikas, O. Leman, J. Hauer, et al., "A comprehensive study of NEMS-based piezoresistive gyroscopes for vestibular implant systems," in 2017 IEEE International Symposium on Inertial Sensors and Systems (INERTIAL), 2017, pp. 1-4.

[39] S. Dellea, P. Rey, and G. Langfelder, "MEMS gyroscopes based on piezoresistive NEMS detection of drive and sense motion," Journal of Microelectromechanical Systems, vol. 26, pp. 13891399, 2017.

[40] A. Kottapalli, C. Tan, M. Olfatnia, J. Miao, G. Barbastathis, and M. Triantafyllou, "A liquid crystal polymer membrane MEMS sensor for flow rate and flow direction sensing applications," Journal of Micromechanics and Microengineering, vol. 21, p. 085006, 2011.

[41] M. Asadnia, S. M. M. Ehteshami, S. H. Chan, and M. E. Warkiani, "Development of a fiber-based membraneless hydrogen peroxide fuel cell," RSC Advances, vol. 7, pp. 40755-40760, 2017.

[42] A. Kottapalli, M. Asadnia, E. Kanhere, M. Triantafyllou, and J. Miao, "Smart skin of selfpowered hair cell flow sensors for sensing hydrodynamic flow phenomena," in 2015 Transducers-2015 18th International Conference on Solid-State Sensors, Actuators and Microsystems (TRANSDUCERS), 2015, pp. 387-390.

[43] A. G. Kottapalli, M. Asadnia, J. Miao, G. Barbastathis, and M. S. Triantafyllou, "A flexible liquid crystal polymer MEMS pressure sensor array for fish-like underwater sensing," Smart Materials and Structures, vol. 21, p. 115030, 2012.

[44] A. G. P. Kottapalli, M. Asadnia, J. M. Miao, G. Barbastathis, and M. S. Triantafyllou, "A flexible liquid crystal polymer MEMS pressure sensor array for fish-like underwater sensing," Smart Materials and Structures, vol. 21, p. 115030, 2012/10/26 2012. 
[45] A. G. P. Kottapalli, C. W. Tan, M. Olfatnia, J. M. Miao, G. Barbastathis, and M. Triantafyllou, "A liquid crystal polymer membrane MEMS sensor for flow rate and flow direction sensing applications," Journal of Micromechanics and Microengineering, vol. 21, Aug 2011.

[46] B. Abbasnejad, W. Thorby, A. Razmjou, D. Y. Jin, M. Asadnia, and M. E. Warkiani, "MEMS piezoresistive flow sensors for sleep apnea therapy," Sensors and Actuators a-Physical, vol. 279, pp. 577585, Aug 2018.

[47] M. Asadnia, A. G. P. Kottapalli, K. D. Karavitaki, M. E. Warkiani, J. Miao, D. P. Corey, et al., "From biological cilia to artificial flow sensors: Biomimetic soft polymer nanosensors with high sensing performance," Scientific reports, vol. 6, p. 32955, 2016.

[48] J. Ten Kate, H. Van Barneveld, and J. Kuiper, "The dimensions and sensitivities of semicircular canals," Journal of Experimental Biology, vol. 53, pp. 501514, 1970.

[49] D. Obrist, "Flow Phenomena in the Inner Ear," Annual Review of Fluid Mechanics, vol. 51, pp. 487510, 2019.

[50] G. E. Grossman, R. J. Leigh, L. Abel, D. J. Lanska, and S. Thurston, "Frequency and velocity of rotational head perturbations during locomotion," Experimental brain research, vol. 70, pp. 470-476, 1988.

[51] M. Asadnia, A. Kottapalli, J. Miao, A. Randles, A. Sabbagh, P. Kropelnicki, et al., "High temperature characterization of PZT $(0.52 / 0.48)$ thin-film pressure sensors," Journal of Micromechanics and Microengineering, vol. 24, p. 015017, 2013.

[52] V. F. Ferrario, C. Sforza, G. Serrao, G. Grassi, and E. Mossi, "Active range of motion of the head and cervical spine: a three-dimensional investigation in healthy young adults," Journal of orthopaedic research, vol. 20, pp. 122-129, 2002.

[53] M. Hitier, M. Hamon, P. Denise, J. Lacoudre, M.-A. Thenint, J.-F. Mallet, et al., "Lateral semicircular canal asymmetry in idiopathic scoliosis: an early link between biomechanical, hormonal and neurosensory theories?," PloS one, vol. 10, p. e0131120, 2015. 

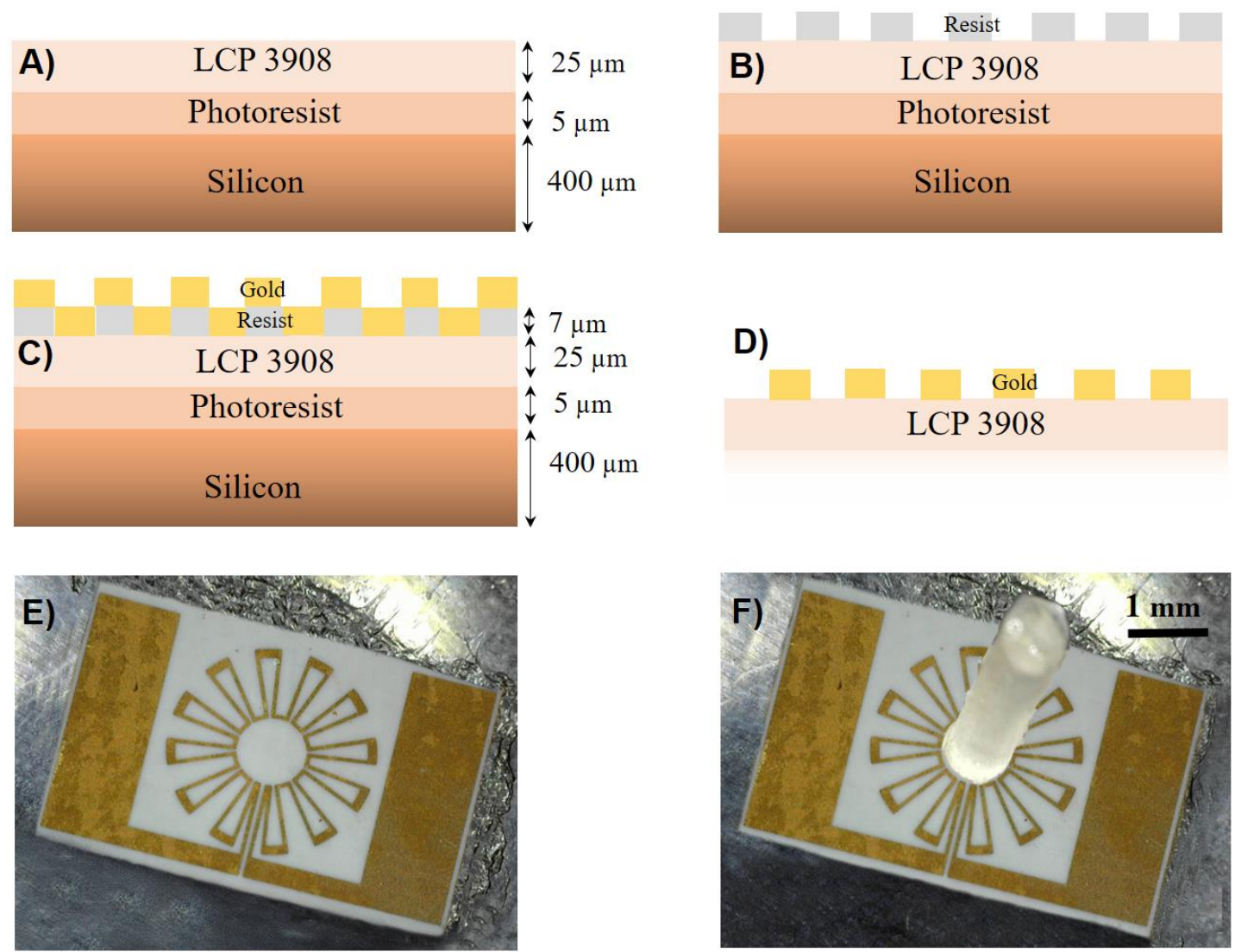

Fig. 1. Overview of the fabrication process for the LCP flow sensor: a) LCP-silicon bonding with a thin layer of photoresist intermediate layer, b) resist patterning, c) $100 \mathrm{~nm}$ gold sputter deposition d) gold lift-off by dissolving the photoresist and removing the silicon and resist, e) completed LCP pressure sensor, f) finalized flow sensor with polymer pillar glued to the LCP pressure sensor

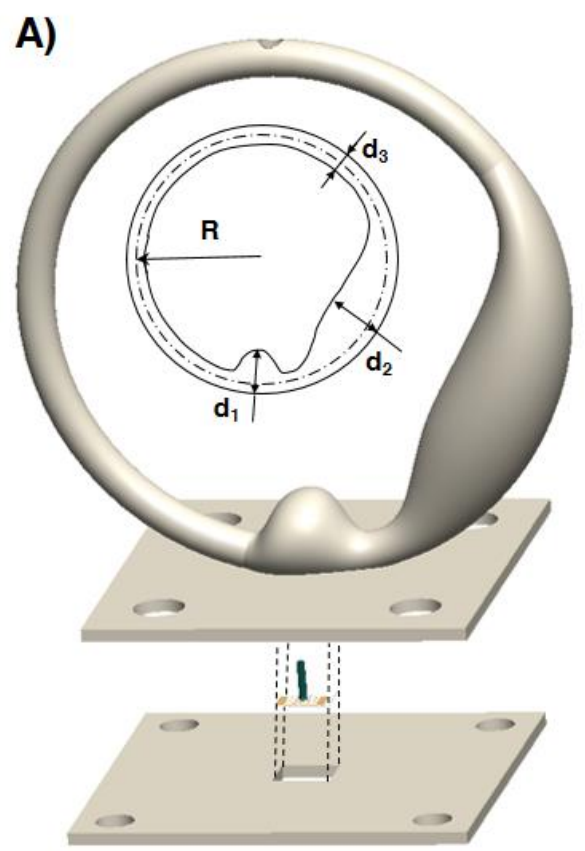

B)

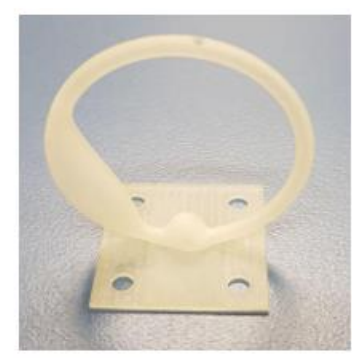

D)

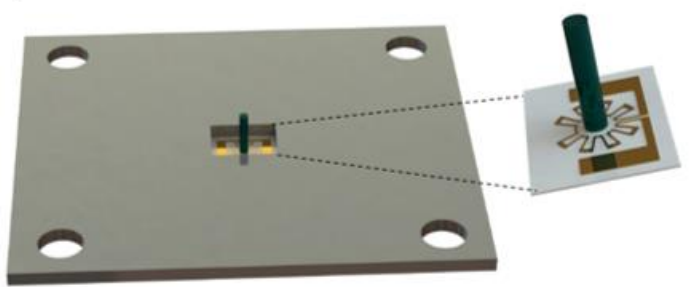

Fig. 2. Sensor fabrication and packaging process: A) Solidworks model comprising the base (sensor holder), flow sensor and LSCC model with its simplified geometry based on morphological data, B) 3D printed model of LSCC, C) Finalized LSCC package with flow sensor embedded in ampulla and mounted on the base, D) magnified view of LCP flow sensor fixed on base. 


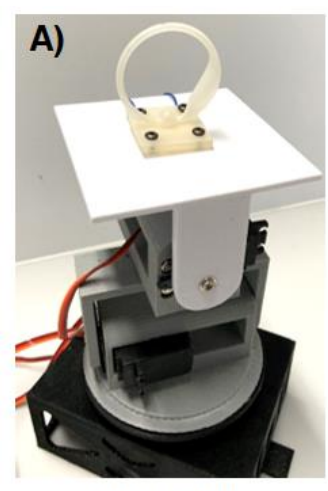

Rotary table

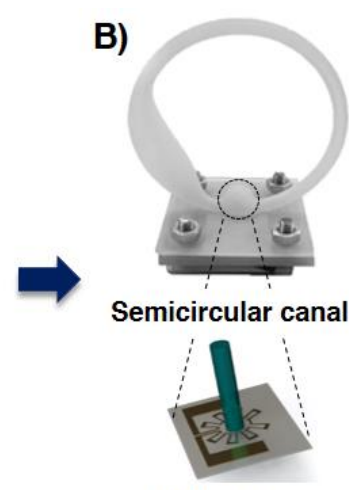

LCP sensor

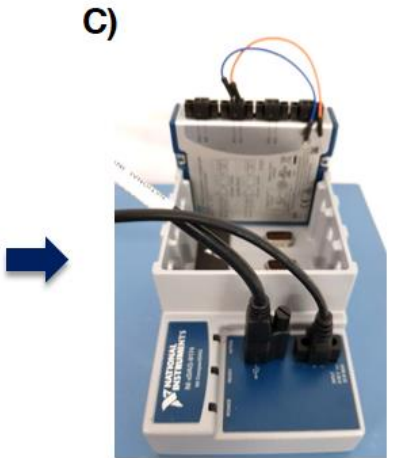

Data acquisition

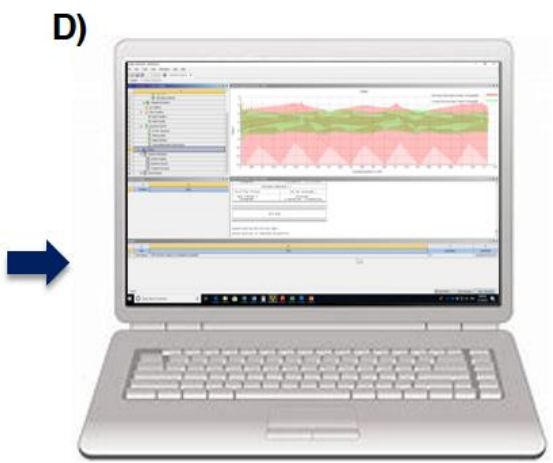

Data analysis

Fig. 3. Overview of the devices used to test and record the LSCC response to head movements. A) LSCC mounted on top pf the rotary table capable of rotation in different directions. B) Flow sensor embedded inside the LSCC. C) Data acquisition unit records analog data from the sensor. D) Laptop used to control table and run LabVIEW Signal Express software for visualization and analysis of the sensor output.

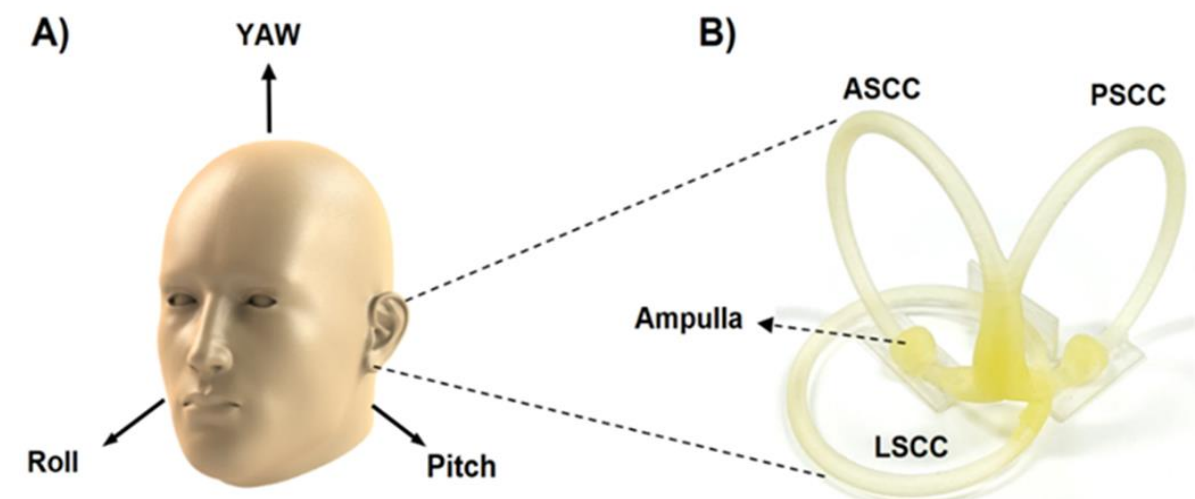

Fig. 4. A) Head rotation in pitch, yaw and roll axes, B) Magnified view of SCCs. Head movement can be decomposed to roll, yaw and pitch axes, each mainly sensed by ASCC (anterior SCC), LSCC (lateral SCC), and PSCC (posterior SCC) respectively. 
A)
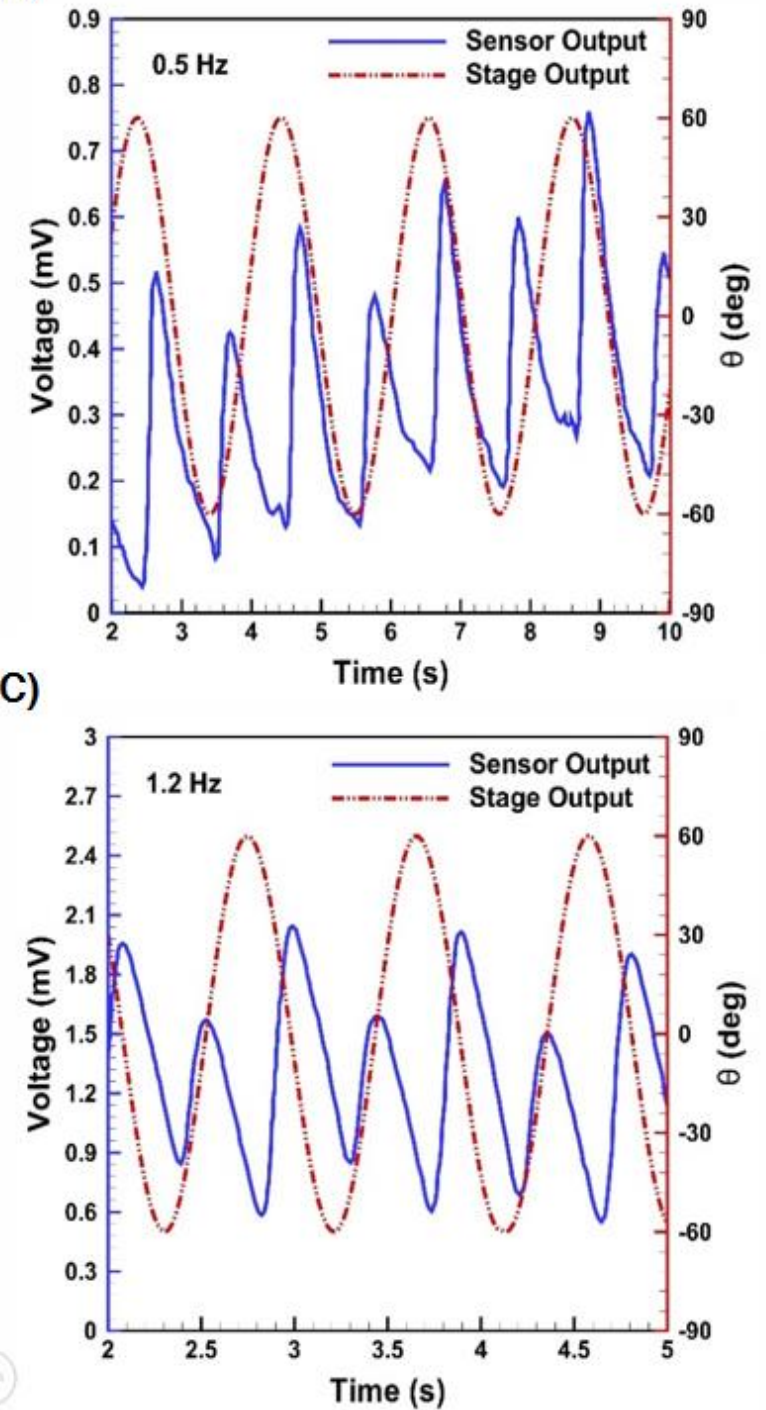

B)
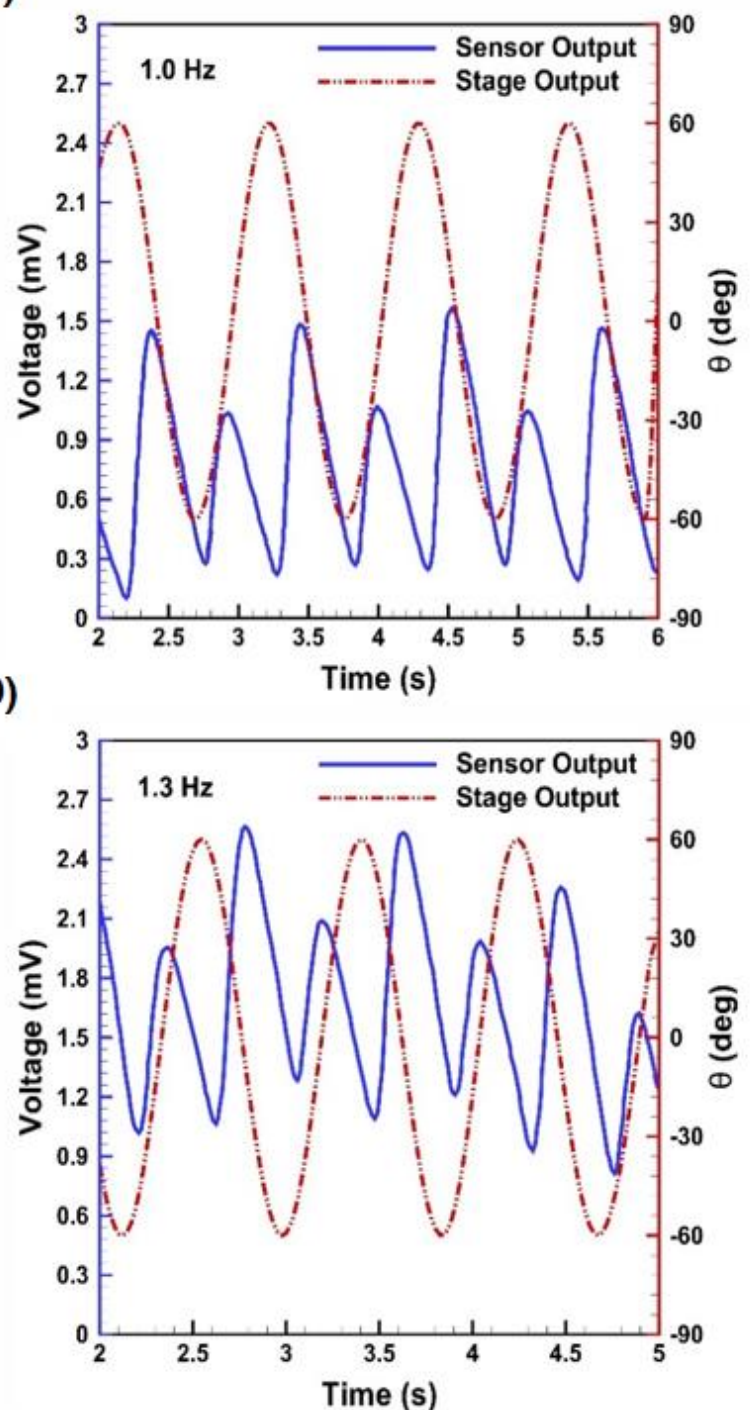

Fig. 5. Sensor- stage output comparison for 60 degree rotation with various frequencies: A) $0.5 \mathrm{~Hz}, \mathrm{~B}) 1 \mathrm{~Hz}, \mathrm{C}) 1.2 \mathrm{~Hz}$, and D) $1.3 \mathrm{~Hz}$. 
A)

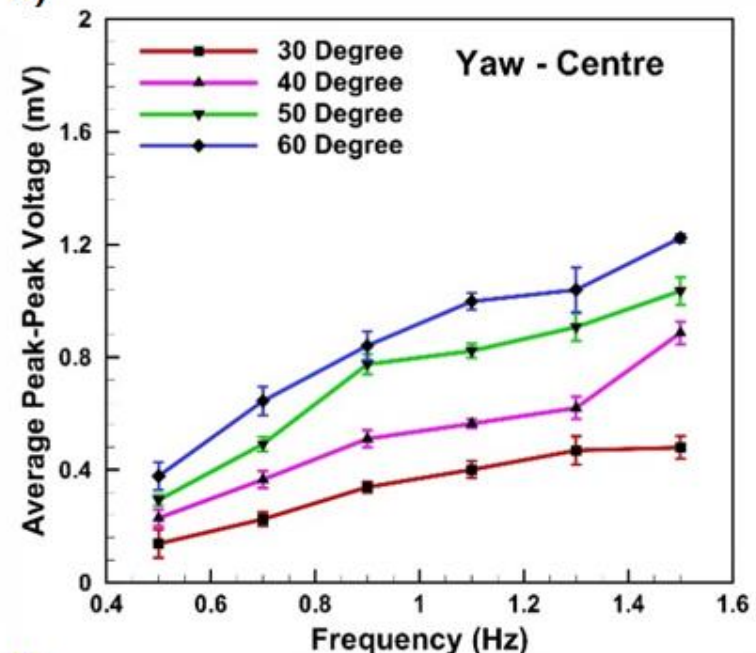

C)

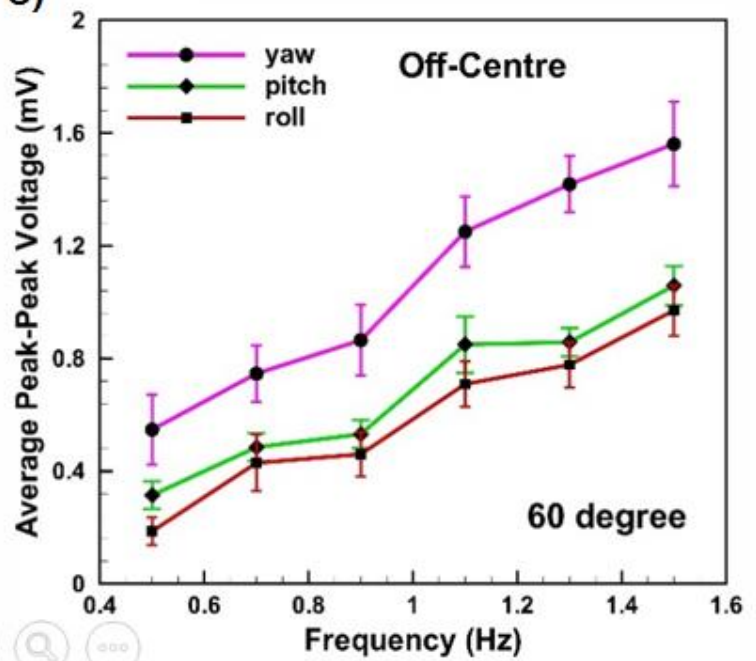

B)

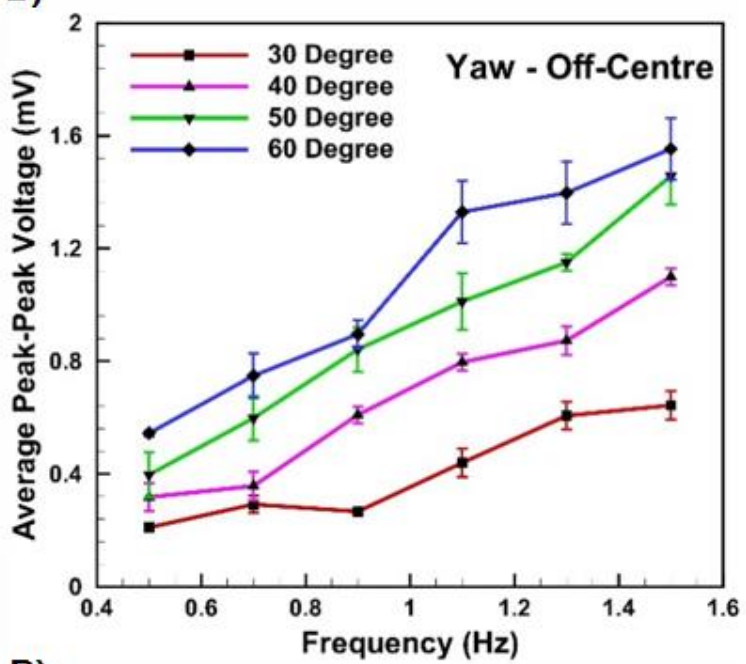

D)

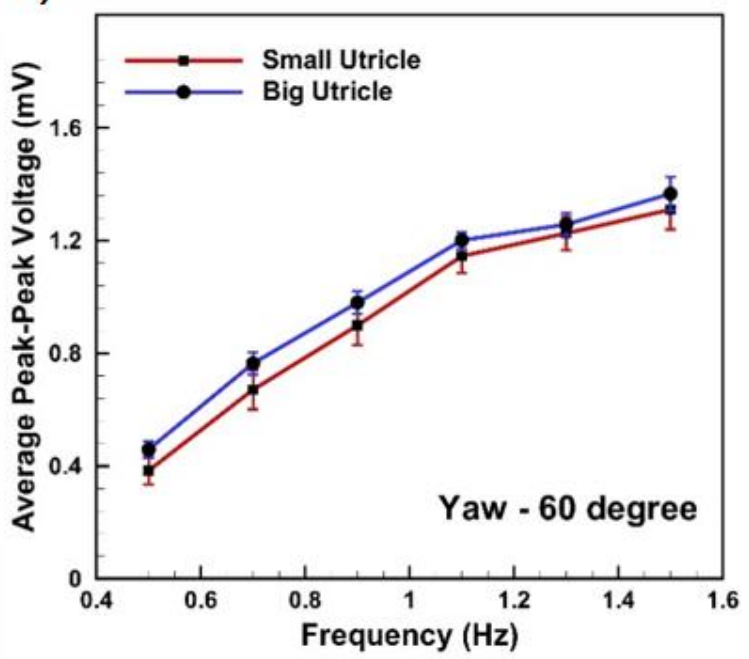

Fig. 6. Average peak-peak voltage of the sensor versus frequency of the rotation for: A) yaw rotation when LSCC is exactly positioned on the centre of rotation, B) yaw rotation for LSCC off-centre position, C) rotation around yaw, roll and pitch axes, D) LSCC with different size of utricle.

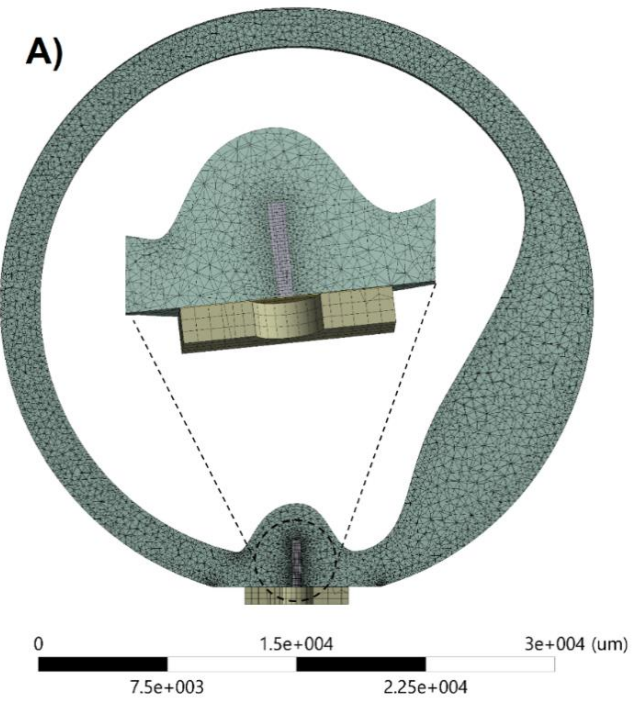

B)

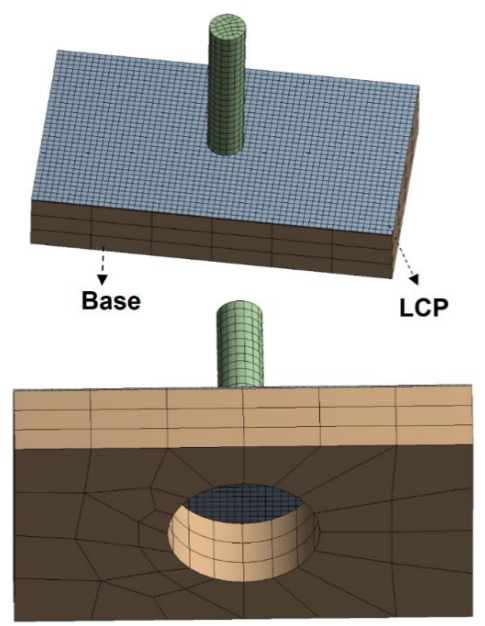

Fig. 7. Geometry and the mesh configuration for fluid solid interaction modelling, A) complete model and the magnified mesh in the vicinity of the sensor. For the semicircular canal tetrahedral mesh is used, B) uniform mesh over the sensor pillar and the LCP, C) bottom view from the sensor base. To model the movement of the LCP, we attached it to a solid base with a hole in its centre that facilitates free movement of the LCP due to the sensor movement. 


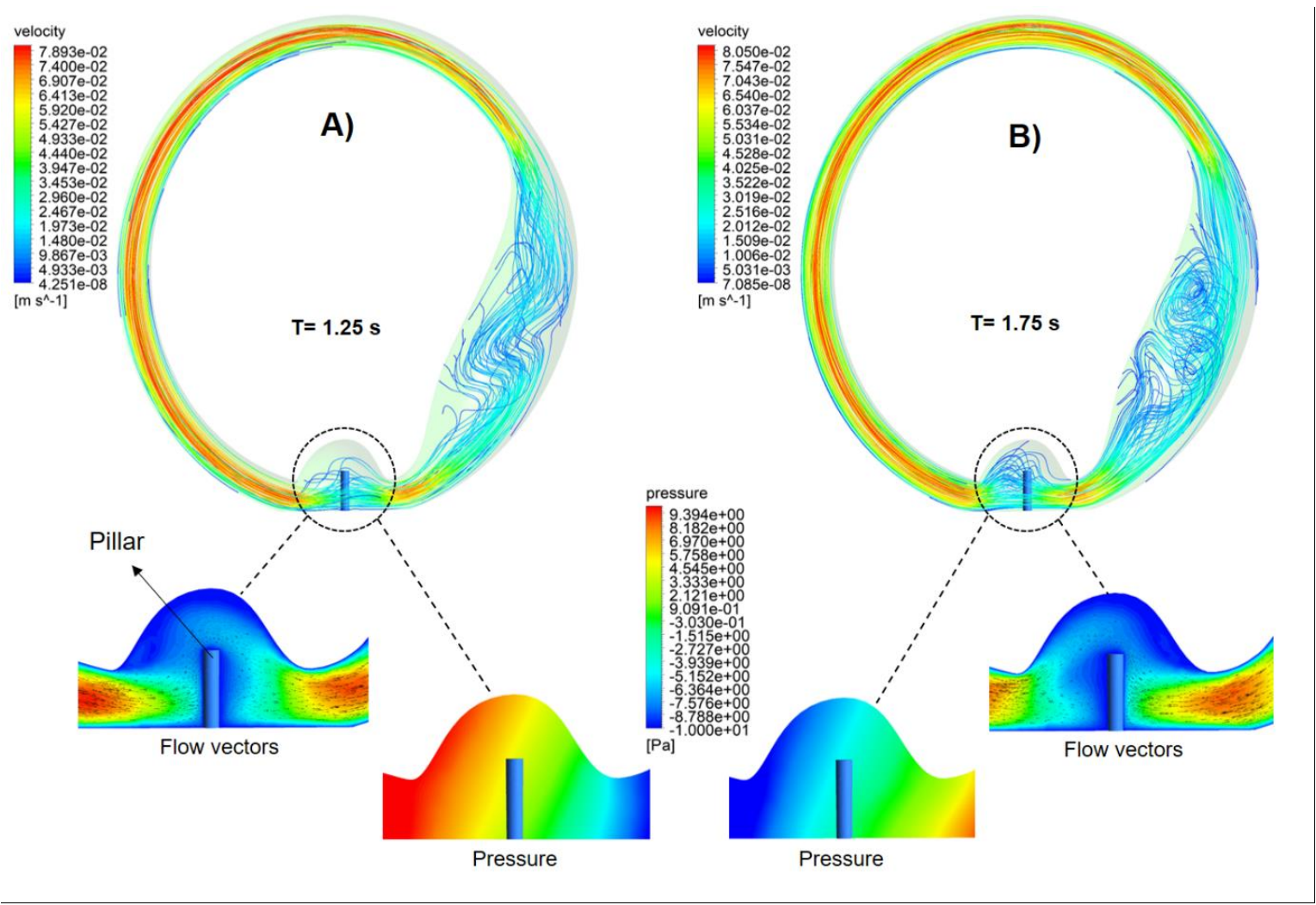

Fig. 8. Streamlines, pressure and velocity contours in LSCC and near the pillar for A) $t=1.25 \mathrm{~s}$ and B) $t=1.75 \mathrm{~s}$. 
A)

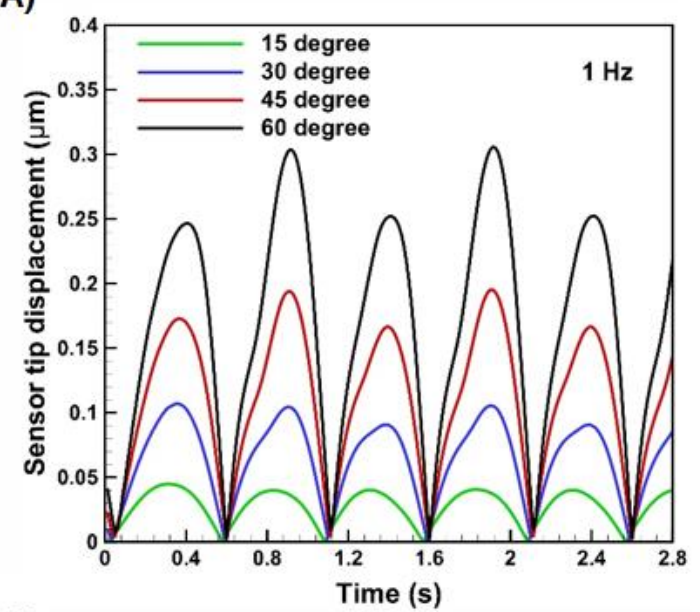

C)

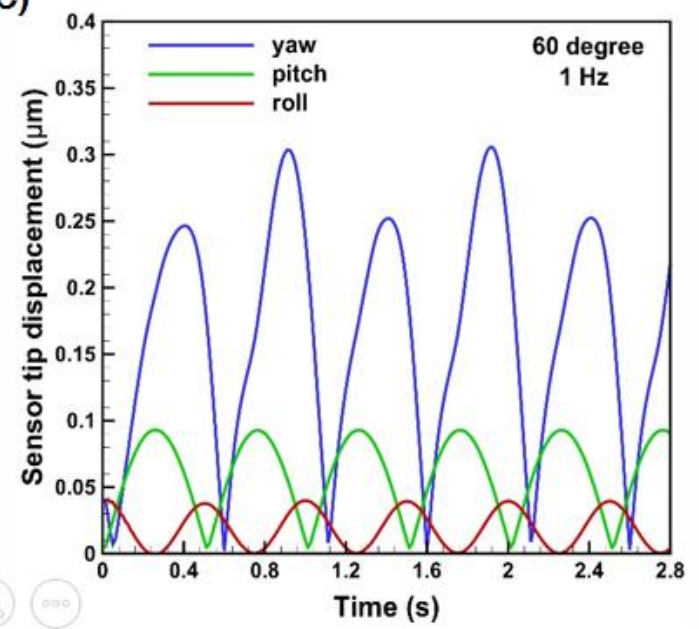

B)
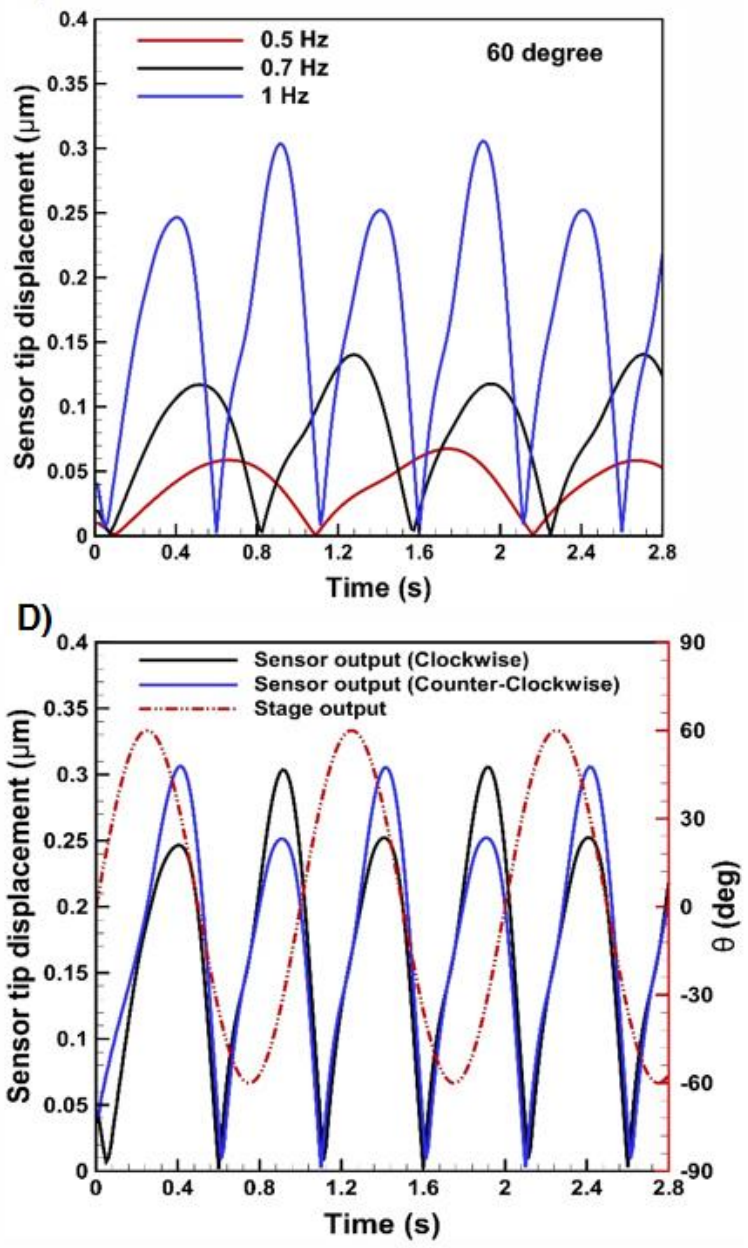

Fig. 9. A) Pillar tip displacement for different rotation angles at $1 \mathrm{~Hz}$ frequency, B) pillar tip displacement for different rotation frequencies at 60 -degree rotation

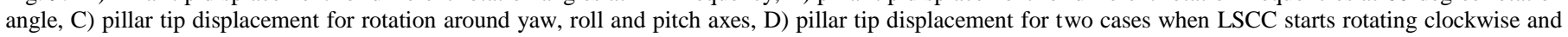
counter clockwise (60-degree rotation and $1 \mathrm{~Hz}$ ).

TABLE I

PHYSICAL PROPERTIES OF LCP BIOMIMETIC HAIR SENSOR

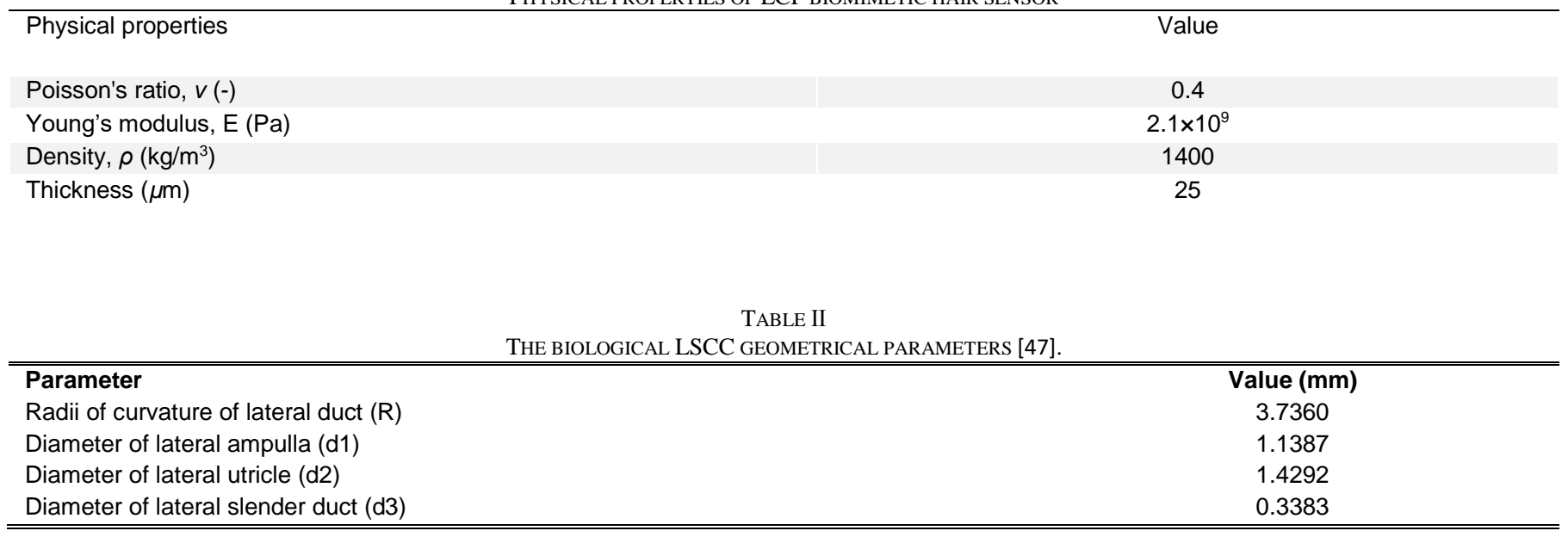




\section{IEEE SENSORS}

TABLE III

CHARACTERISTIC MOVEMENT LIMITS OF THE HEAD IN MALES AND FEMALES BASED ON DESCRIPTIVE STATISTICS (MEAN AND STANDARD DEVIATION (S. D.))[52]

\begin{tabular}{lcccc}
\hline \hline \multirow{2}{*}{ Limit of Rotation } & \multicolumn{2}{c}{ Female } & \multicolumn{2}{c}{ Male } \\
& Mean (degree) & S. D. & Mean (degree) & S. D. \\
\hline \hline Neck Rotation (right) & 81.8 & 7.2 & 79.8 & 7.6 \\
Neck Rotation (left) & 80.1 & 7.7 & 75.3 & 8.2 \\
Neck Flexion (forward) & 58.5 & 9.7 & 60.4 & 12.1 \\
Neck Extension (backward) & 77.5 & 13.2 & 69.9 & 12.7 \\
Neck Lateral Bend (left) & 45.5 & 7.6 & 36.3 & 8.0 \\
Neck Lateral Bend (right) & 45.3 & 9.5 & 40.9 & 8.3 \\
\hline \hline
\end{tabular}

